\title{
Migrants, soins au corps et soins psychiques
}

\author{
Abdelhak Elghezouani \\ Psychologue, Consultation Psychothérapeutique pour Migrants, Association Appartenances Vaud
}

Dans le travail clinique avec des patients migrants, en particulier ceux ayant vécu des violences politiques, les professionnels et les dispositifs de soins peuvent se trouver confrontés à des manifestations cliniques atypiques ou «résistantes», psychiatriques ou psychosomatiques. Les enjeux épistémologiques, théoriques et techniques posés par ce type de clinique ont fait et font encore débat. Mais des disciplines telles que l'anthropologie culturelle, l'anthropologie psychiatrique et médicale ont apporté des éclairages permettant de prendre en compte certaines expressions de l'altérité et de la diversité caractérisant les facteurs et les expressions de la souffrance psychique. Dans une démarche en complémentarité avec nos partenaires du réseau médico-social, nous proposons un dispositif d'inspiration ethnopsychiatrique lorsque la "chaîne de soins" se trouve à risque d'être rompue ou lorsqu'il s'agit de la rétablir, de la réorienter voire de l'initier.

\section{Pluridisciplinarités}

L’anthropologie médicale a apporté plusieurs concepts permettant d'améliorer l'intercompréhension et la relation entre un soignant dit "occidental» et un patient dit «non occidental». La question des rapports entre culture et psychisme, entre santé mentale et culture a, elle, bénéficié du développement quasi simultané de l'anthropologie et de la psychanalyse, à

\section{Physische und psychische Gesundheitsversorgung von Migranten}

In diesem Artikel stellen wir ein psychotherapeutisches Modell vor, das wir auf der Grundlage unserer Arbeit mit Migranten entwickelt haben, die Opfer politischer Gewalt geworden sind. Das Modell ist Teil der psychotherapeutischen Beratung ${ }^{*}$, die unser Verein "Association Appartenances» für Migranten anbietet. Der Verein ist für Migranten in vielerlei Hinsicht tätig. Sie haben uns dazu bewegt, unser therapeutisches Angebot als einen Ort des Übergangs zwischen ihnen und der aufnehmenden Gesellschaft, zwischen ihrer Gegenwart und ihrer Vergangenheit zu konzipieren. Unser Modell berücksichtigt die unterschiedlichen Dimensionen und Gegebenheiten, anhand derer die Betroffenen sich selbst definieren. Ein Patient wird von mehreren Ko-Therapeuten betreut, die entsprechend der komplementaristischen Methode auf unterschiedliche Dimensionen seiner Problematik eingehen. Diese Dimensionen können kultureller, sozialer oder psychologischer Art sein. la fin du XIXe siècle. Dans le champ spécifique de la santé mentale, nous avons assisté à l'éclosion de disciplines et d'appellations telles que «Transcultural Psychiatry", "Crosscultural Psychiatry» ou "Psychiatrie Transculturelle», «Ethnopsychiatrie» et «Ethnopsychanalyse».

Les apports mutuels entre psychiatrie et anthropologie concernent aussi la médecine somatique et la psychosomatique à travers, par exemple, les diverses conceptions culturelles du corps, de ses parties et de leurs fonctions symboliques, ou à travers les expressions psychosomatiques dans lesquelles agissent des représentations culturelles et mentales. En effet, dans certaines circonstances, le corps devient le lieu même où s'inscrit une partie de la vie psychique lorsque cette dernière se trouve privée de la capacité de représentation et de symbolisation.

En outre, concernant les migrants, les acculturations produites par la rencontre avec une certaine modernité dans les pays d'origine et par le contact avec la société d'accueil occidentale et ses diverses institutions et professionnels produisent des effets qu'il s'agirait de prendre en charge dans des «lieux de transitionnalité» [3].

\section{Altérité ou étrangeté?}

Hypocondrie, troubles fonctionnels, maladies psychosomatiques donnent lieu à des tableaux cliniques qui sont autant d'interfaces entre des éléments culturels et sociaux, et des mouvements psychiques provoquées par des causes diverses. La situation transculturelle peut ainsi se trouver saturée, parfois paralysée, par les plaintes somatiques mises en avant, dans un contexte d'inhibition et de limitation de l'expression verbale émotionnelle allant au-delà de difficultés purement linguistiques. Ainsi, «les aspects somatiques de la souffrance peuvent avoir une fonction défensive comme cela est fréquemment le cas chez les demandeurs d'asile, victimes de traumatismes psychiques graves» [1].

A notre sens, l'explication serait plus à rechercher du côté de mécanismes psychopathologiques, physio- 
logiques et neurophysiologiques que de celui des cultures, tant l'impact des violences réelles et symboliques et de la précarité peut être déterminant. Si la culture intervient, c'est avec une majuscule, à travers le «Travail de la Culture» [4] sur le psychisme. Les items culturels servant d'objets et d'écrans de projection dans le cadre de mouvements psychiques. Il convient aussi de se tourner vers les apports de la psychosomatique analytique [7] face à des plaintes somatiques et à des mouvements psychiques fréquemment retrouvés chez des patients en «exil culturel» et/ou avec un vécu traumatique ayant perdu leurs capacités de symbolisation et d'élaboration.

\section{Métissages}

Certains traumatismes peuvent provoquer la brutale apparition d'un tableau psychopathologique à l'occasion d'un accident ou d'une maladie entraînant plaintes et incapacités. Ces événements semblant entrés en résonance avec un traumatisme antérieur incorporé dans le psychisme du patient et devenu un corps psychique mortifère que J. Guyotat appelle "chrone» [5].

Ce type de tableaux cliniques s'accompagne parfois d'un «métissage» des symptomatologies. Le patient pouvant développer de manière alternée et parfois paradoxale des symptômes ou une étiologie le reliant à sa culture d'origine et d'autres conformes à la biomédecine occidentale. Ce métissage est bien un phénomène moderne, conséquence d'une forme d'acculturation, que des psychiatres comme D. Schurmans ont identifié dans les pays d'origine même [8]. Il faut donc aussi considérer que les migrants proviennent de sociétés plus ou moins acculturées, composites et en transition. La nécessité de métisser nos dispositifs de soins devient dès lors plus cruciale en présence de tableaux cliniques résistants ou atypiques.

\section{Une approche complémentariste}

Comment dès lors introduire une dimension culturelle et socio-historique dans le traitement de patients migrants présentant, par exemple, un tableau clinique où se mêlent des plaintes somatiques récurrentes et des symptômes psychiatriques sévères et invalidants ayant dérouté maints intervenants et motivé de multiples interventions diagnostiques et thérapeutiques, tant somatiques que psychiatriques? A notre sens, ces problématiques doivent être traitées, de manière complémentariste, sur trois niveaux: anthropologique et culturel, sociopolitique (les conditions matérielles de vie ici et dans le pays d'origine sont autant de facteurs entrant en ligne de compte dans la précipitation, le maintien ou la péjoration des troubles) et psychologique. Ce dernier niveau nécessitant de prendre en compte les pertes de soi-même, des autres et du sens, et de favoriser la capacité à créer un nouveau sens à l'existence et de nouvelles valeurs [6]. Le niveau anthropologique et culturel insistera sur l'expérience migratoire et d'acculturation à partir des différences et des contrastes entre ici et là-bas, entre avant et après, entre soi et les autres. Enfin, le niveau socio-historique ne peut être évité car les réalités sociales constituent autant de coordonnées à partir desquelles l'individu se définit. La méthodologie complémentariste implique donc plusieurs «lectures» non simultanées, sachant aussi qu'aucune ne peut à elle seule circonscrire la question.

L'enjeu est de travailler sur ces multiples niveaux, de manière non simultanée mais systématique, précisément parce que les dimensions anthropologique, culturelle, sociopolitique et psychique se trouvent circulairement et dialectiquement liées. Dans ce paradigme, nous pouvons alors intervenir sur la réalité externe et sur la réalité interne, et non cloisonner ces deux domaines pourtant indissociables en termes de vécus subjectifs. Nous pouvons aussi inclure un tiers, par exemple un interprète ou tout autre professionnel, et nous pouvons assumer le rôle de passeur entre le patient et une réalité matérielle externe, ici ou là-bas, qui lui est incompréhensible ou insupportable.

\section{Un dispositif d'inspiration ethno- psychiatrique}

«Dispositif d'Inspiration Ethnopsychiatrique» (DIE) signifie qu'il s'agit d'un groupe de co-thérapeutes agissant dans le cadre d'un dispositif thérapeutique inspiré de la méthode complémentariste de G. Devereux [2]. Coordonnés par un thérapeute principal, les co-thérapeutes reçoivent un patient et éventuellement des accompagnants pour une ou plusieurs séances de deux à trois heures. Le patient peut être adressé par un professionnel du réseau ou par un thérapeute de notre consultation. Etant donné le nombre de thérapeutes impliqués et la durée des séances, ce type de dispositif ne peut être entièrement financé par les assurances. Le but de ce dispositif est de fournir un éclairage nouveau et original à une problématique de santé ayant posé problème à d'autres professionnels ou à risque de ne pas être prise en charge ou de faire l'objet d'une rupture. La plupart du temps, une séance suffit à réorienter la prise en charge ou à susciter la mise en place d'une thérapie mieux ciblée sur certains éléments décisifs. 
Par sa dynamique et par ses règles de fonctionnement, le dispositif vise des objectifs qui ne peuvent être atteints en dehors de sa méthodologie et de ses modalités spécifiques. Ces objectifs sont la découverte, la mise à plat et en dialogue de représentations et de motivations variées. Celles-ci peuvent souvent être concurrentes ou non exprimées et aboutir à une souffrance psychique, à des dysfonctionnements voire à des conflits. Le dépassement de cette conflictualité permet par la suite de nouvelles constructions mentales, relationnelles et fonctionnelles.

Le DIE se caractérise par la pluralité des protagonistes et donc des relations intersubjectives, et par la pluralité des processus favorisés, observés et analysés. Les indications sont constituées par les problématiques de santé permettant une lecture ou une intervention mobilisant des représentations et des dynamiques "culturelles» et socio-historiques influençant l'expression symptomatologique ou invoquées comme étiologies.

Il est axé sur l'expression, la figuration, la mise en significations, la dialectique des vécus, des ressentis, des significations individuelles et collectives. Il est aussi axé sur la prise de conscience du processus luimême et sur la verbalisation de celle-ci. L'accent étant mis sur ce qui se passe dans l'ici et maintenant. Il s'agit en fait de développer des faits, d'exprimer des vécus et des ressentis, d'échanger et d'élaborer autour de diverses significations et, enfin, de rendre compte du processus et d'«historiciser» ainsi la rencontre. Ce dispositif ponctue ou initie une prise en charge. Il représente un moment dans la relation entre plusieurs protagonistes, dont il devient lui-même, un moment, un protagoniste.

En outre, il s'agit d'y invoquer et d'y analyser le «Travail de la Culture», en tant que condition, cadre et support de la vie psychique, et non pas seulement d'y convoquer des items culturels. La dynamique de l'acculturation passée et présente est aussi mobilisée et élaborée. L'altérité à soi-même et aux autres de chacun des protagonistes peut ainsi être sollicitée, interpellée et interrogée. Les «lectures» culturelles idiosyncrasiques fournies par le patient et par les co-thérapeutes permettent de mobiliser, de retrouver et de construire les contenants et les contenus culturels groupaux et individuels ayant fait défaut au patient ou permettant d'agir sur la problématique dans un contexte d'acculturation souvent violente, soudaine, radicale et non reconnue. Elles portent sur ce qui fait problème ou symptôme et sont ainsi contextualisées et problématisées. La culture est ici invoquée en tant qu'elle fonde l'identité et en tant que cette identité se trouve mise à mal pour certains migrants. Mais nous tenons compte aussi du fait que la culture peut être instrumentalisée dans des mouvements défensifs et projectifs autant chez les migrants que chez les professionnels.

\section{Conclusion}

Nous avons voulu rendre compte d'une conceptualisation se trouvant à la base de nos prises en charge de migrants précarisés et victimes de violences politiques. Ces patients nous ont amenés à concevoir nos dispositifs ainsi que nos pratiques thérapeutiques comme des lieux transitionnels entre ceux-ci et la société d'accueil, entre leur présent et leur passé, prenant en compte, dans une démarche complémentariste, les multiples dimensions et les multiples données par lesquelles ils se définissent eux-mêmes. Lorsque l'individu exprime une souffrance dont les déterminants sont multiples, politiques, sociaux, culturels; lorsqu'à l'altérité culturelle viennent s'ajouter la précarité sociale et les séquelles de violences; lorsque les tableaux cliniques s'entremêlent, se brouillent et se complexifient mêlant pathologies et plaintes somatiques à des troubles psychiatriques; lorsque les problématiques deviennent de parfaites énigmes, les modalités de prises en charge doivent se métisser et s'exposer à la multidisciplinarité.

\section{Références}

1 Baubet T, Moro MR (éds). Psychiatrie et migrations. (Rapport de psychiatrie au congrès de psychiatrie et de neurologie de langue française.) Paris: Masson; 2003.

2 Devereux G. Ethnopsychanalyse complémentariste. Paris: Flammarion; 1972 (réédition 1985).

3 Douville O, Galap J. Santé mentale des migrants et des réfugiés en France. Encyclopédie Médico-Chirurgicale, Psychiatrie, 37-880-A-10.

4 Freud S. Malaise dans la civilisation. Trad. Weil A. Paris: Payot; 2010.

5 Guyotat J. Traumatisme et lien de filiation. In: Dialogue, $n^{\circ} 168$, 2005/2:15-24.

6 Métraux JC. Deuils collectifs et création sociale. La Dispute, 2004.

7 Pirlot G. Psychopathologie et psychosomatique psychanalytiques. In: Comprendre et traiter les situations interculturelles. Approches psychodynamiques et psychanalytiques (Guerraoui Z, Pirlot G, dir.). De Boek Supérieur, 2011.

8 Schurmans D. Le diable et le bon sens. Paris: L’Harmattan; 1994. 